\title{
LOGARITHMIC COEFFICIENTS FOR CERTAIN SUBCLASSES OF CLOSE-TO-CONVEX FUNCTIONS
}

\author{
U. PRANAV KUMAR AND A. VASUDEVARAO
}

\begin{abstract}
Let $\mathcal{S}$ denote the class of functions analytic and univalent (i.e. oneto-one) in the unit disk $\mathbb{D}=\{z \in \mathbb{C}:|z|<1\}$ normalized by $f(0)=0=f^{\prime}(0)-1$. The logarithmic coefficients $\gamma_{n}$ of $f \in \mathcal{S}$ are defined by $\log \frac{f(z)}{z}=2 \sum_{n=1}^{\infty} \gamma_{n} z^{n}$. In the present paper, we determine the sharp upper bounds for $\left|\gamma_{1}\right|,\left|\gamma_{2}\right|$ and $\left|\gamma_{3}\right|$ when $f$ belongs to some familiar subclasses of close-to-convex functions.
\end{abstract}

\section{INTRODUCTION}

Let $\mathbb{D}:=\{z \in \mathbb{C}:|z|<1\}$ denote the unit disk in the complex plane $\mathbb{C}$. A single-valued function $f$ is said to be univalent in a domain $\Omega \subseteq \mathbb{C}$ if it never takes the same value twice, that is, if $f\left(z_{1}\right)=f\left(z_{2}\right)$ for $z_{1}, z_{2} \in \Omega$ then $z_{1}=z_{2}$. Let $\mathcal{A}$ denote the class of analytic functions $f$ in $\mathbb{D}$ normalized by $f(0)=0=f^{\prime}(0)-1$. If $f \in \mathcal{A}$ then $f(z)$ has the following representation

$$
f(z)=z+\sum_{n=2}^{\infty} a_{n} z^{n} .
$$

Let $\mathcal{S}$ denote the class of univalent functions in $\mathcal{A}$. A domain $\Omega \subseteq \mathbb{C}$ is said to be a starlike domain with respect to a point $z_{0} \in \Omega$ if the line segment joining $z_{0}$ to any point in $\Omega$ lies in $\Omega$. If $z_{0}$ is the origin then we say that $\Omega$ is a starlike domain. A function $f \in \mathcal{A}$ is said to be a starlike function if $f(\mathbb{D})$ is a starlike domain. We denote by $\mathcal{S}^{*}$ the class of starlike functions $f$ in $\mathcal{S}$. It is well-known that [4] a function $f \in \mathcal{A}$ is in $\mathcal{S}^{*}$ if and only if

$$
\operatorname{Re}\left(\frac{z f^{\prime}(z)}{f(z)}\right)>0 \quad \text { for } z \in \mathbb{D} \text {. }
$$

A domain $\Omega$ is said to be convex if it is starlike with respect to each point of $\Omega$. A function $f \in \mathcal{A}$ is said to be convex if $f(\mathbb{D})$ is a convex domain. We denote the class of convex univalent functions in $\mathbb{D}$ by $\mathcal{C}$. A function $f \in \mathcal{A}$ is in $\mathcal{C}$ if and only if

$$
\operatorname{Re}\left(1+\frac{z f^{\prime \prime}(z)}{f^{\prime}(z)}\right)>0 \quad \text { for } z \in \mathbb{D} \text {. }
$$

It is well-known that $f \in \mathcal{C}$ if and only if $z f^{\prime} \in \mathcal{S}^{*}$.

File: Pranav'Vasu'P1`July23`2016 final'Submitted.tex, printed: 2016-7-26, 4.07

2010 Mathematics Subject Classification. Primary 30C45, 30C50.

Key words and phrases. Analytic, univalent, starlike, convex and close-to-convex functions, coefficient estimates, logarithmic coefficients. 
A function $f \in \mathcal{A}$ is said to be close-to-convex (having argument $\alpha \in(-\pi / 2, \pi / 2)$ ) with respect to $g \in \mathcal{S}^{*}$ if

$$
\operatorname{Re}\left(e^{i \alpha} \frac{z f^{\prime}(z)}{g(z)}\right)>0 \quad \text { for } z \in \mathbb{D} \text {. }
$$

We denote the class of all such functions by $\mathcal{K}_{\alpha}(g)$. Let

$$
\mathcal{K}(g):=\bigcup_{\alpha \in(-\pi / 2, \pi / 2)} \mathcal{K}_{\alpha}(g) \text { and } \mathcal{K}_{\alpha}:=\bigcup_{g \in \mathcal{S}^{*}} \mathcal{K}_{\alpha}(g)
$$

be the classes of close-to-convex functions with respect to $g$ and close-to-convex functions with argument $\alpha$, respectively. Let

$$
\mathcal{K}:=\bigcup_{\alpha \in(-\pi / 2, \pi / 2)} \mathcal{K}_{\alpha}=\bigcup_{g \in \mathcal{S}^{*}} \mathcal{K}(g)
$$

denote the class of close-to-convex functions in $\mathcal{A}$. It is well-known that every closeto-convex function is univalent in $\mathbb{D}[10]$. A domain $\Omega \subseteq \mathbb{C}$ is said to be linearly accessible if its complement is the union of a family of non-intersecting half-lines. A function $f \in \mathcal{S}$ whose range is linearly accessible is called a linearly accessible function. Kaplan's theorem [10] makes it seem plausible that the class of linearly accessible family and the class $\mathcal{K}$ coincide. In fact, Lewandowski [11] has observed that the class $\mathcal{K}$ is the same as the class of linearly accessible functions introduced by Biernacki [2] in 1936. In 1962, Bielecki and Lewandowski [1] proved that every function in the class $\mathcal{K}$ is linearly accessible.

Let $\mathcal{P}$ denote the class of analytic functions $h(z)$ of the form

$$
h(z)=1+\sum_{n=1}^{\infty} c_{n} z^{n}
$$

such that $\operatorname{Re} h(z)>0$ in $\mathbb{D}$. To prove our main results we need the following results.

Lemma 1.3. [12] Let $h \in \mathcal{P}$ be of the form (1.2). Then

$$
\begin{aligned}
& 2 c_{2}=c_{1}^{2}+x\left(4-c_{1}^{2}\right) \\
& 4 c_{3}=c_{1}^{3}+2\left(4-c_{1}^{2}\right) c_{1} x-c_{1}\left(4-c_{1}^{2}\right) x^{2}+2\left(4-c_{1}^{2}\right)\left(1-|x|^{2}\right) t .
\end{aligned}
$$

for some complex valued $x$ and $t$ with $|x| \leq 1$ and $|t| \leq 1$.

Lemma 1.4. [14, pp 166] Let $h \in \mathcal{P}$ be of the form (1.2). Then

$$
\left|c_{2}-\frac{c_{1}^{2}}{2}\right| \leq 2-\frac{\left|c_{1}\right|^{2}}{2} \text {. }
$$

The inequality is sharp for functions $L_{t, \theta}(z)$ of the form

$$
L_{t, \theta}(z)=t\left(\frac{1+e^{i \theta} z}{1-e^{i \theta} z}\right)+(1-t)\left(\frac{1+e^{i 2 \theta} z^{2}}{1-e^{i 2 \theta} z^{2}}\right) .
$$

Lemma 1.5. 13] Let $h \in \mathcal{P}$ be of the form (1.2) and $\mu$ be a complex number. Then

$$
\left|c_{2}-\mu c_{1}^{2}\right| \leq 2 \max \{1,|2 \mu-1|\} .
$$

The result is sharp for the functions given by $p(z)=\frac{1+z^{2}}{1-z^{2}}$ and $p(z)=\frac{1+z}{1-z}$. 
Given a function $f \in \mathcal{S}$, the coefficients $\gamma_{n}$ defined by

$$
\log \frac{f(z)}{z}=2 \sum_{n=1}^{\infty} \gamma_{n} z^{n}
$$

are called the logarithmic coefficients of $f(z)$. The logarithmic coefficients are central to the theory of univalent functions for their role in the proof of Bieberbach conjecture. Milin conjectured that for $f \in \mathcal{S}$ and $n \geq 2$,

$$
\sum_{m=1}^{n} \sum_{k=1}^{m}\left(k\left|\gamma_{k}\right|^{2}-\frac{1}{k}\right) \leq 0
$$

Since Milin's conjecture implies Bieberbach conjecture, in 1985, De Branges proved Milin conjecture to give an affirmative proof of the Bieberbach conjecture [3].

By differentiating (1.6) and equating coefficients we obtain

$$
\begin{aligned}
\gamma_{1} & =\frac{1}{2} a_{2} \\
\gamma_{2} & =\frac{1}{2}\left(a_{3}-\frac{1}{2} a_{2}^{2}\right) \\
\gamma_{3} & =\frac{1}{2}\left(a_{4}-a_{2} a_{3}+\frac{1}{3} a_{2}^{3}\right) .
\end{aligned}
$$

It is evident from (1.7) that $\left|\gamma_{1}\right| \leq 1$ if $f \in \mathcal{S}$. An application of Fekete-Szegö inequality [4, Theorem 3.8] in (1.8) yields the following sharp estimate

$$
\left|\gamma_{2}\right| \leq \frac{1}{2}\left(1+2 e^{-2}\right)=0.635 \ldots \quad \text { for } f \in \mathcal{S} .
$$

The problem of finding the sharp upper bound for $\left|\gamma_{n}\right|$ for $f \in \mathcal{S}$ is still open for $n \geq 3$. The sharp upper bounds for modulus of logarithmic coefficients are known for functions in very few subclasses of $\mathcal{S}$. For the Koebe function $k(z)=z /(1-z)^{2}$, the logarithmic coefficients are $\gamma_{n}=1 / n$. Since the Koebe function $k(z)$ plays the role of extremal function for most of the extremal problems in the class $\mathcal{S}$, it is expected that $\left|\gamma_{n}\right| \leq \frac{1}{n}$ holds for functions in the class $\mathcal{S}$. However, this is not true in general. Indeed, there exists a bounded function $f$ in the class $\mathcal{S}$ with logarithmic coefficients $\gamma_{n} \neq O\left(n^{-0.83}\right)$ (see [4, Theorem 8.4]). A simple exercise shows that $\left|\gamma_{n}\right| \leq 1 / n$ for functions in $\mathcal{S}^{*}$ and the equality holds for the Koebe function. Consequently, attempts have been made to find bounds for logarithmic coefficients for close-to-convex functions in the unit disk $\mathbb{D}$. Elhosh [6] attempted to extend the result $\left|\gamma_{n}\right| \leq 1 / n$ to the class $\mathcal{K}$. However Girela [8] pointed out an error in the proof and proved that for every $n \geq 2$ there exists a function $f$ in $\mathcal{K}$ such that $\left|\gamma_{n}\right| \geq 1 / n$. Ye [19] provided an estimate for $\left|\gamma_{n}\right|$ for functions $f$ in the class $\mathcal{K}$, showing that $\left|\gamma_{n}\right| \leq A n^{-1} \log n$ where $A$ is a constant. The sharp inequalities are known for sums involving logarithmic coefficients (see [4, 5]). For $f \in \mathcal{S}$, Roth [17] proved the following sharp inequality

$$
\sum_{n=1}^{\infty}\left(\frac{n}{n+1}\right)^{2}\left|\gamma_{n}\right|^{2} \leq 4 \sum_{n=1}^{\infty}\left(\frac{n}{n+1}\right)^{2} \frac{1}{n^{2}}=\frac{2 \pi^{2}-12}{3} .
$$


Recently, it has been proved that $\left|\gamma_{3}\right| \leq 7 / 12$ for functions in the class $\mathcal{K}_{0}$ with the additional assumption that the second coefficient of the corresponding starlike function $g(z)$ is real [18]. However this bound is not sharp. Enough emphasis cannot be laid on this fact as it highlights nature of complexity involved in obtaining the sharp upper bound for $\left|\gamma_{3}\right|$. More recently Firoz and Vasudevarao [7] improved the bound on $\left|\gamma_{3}\right|$ by proving $\left|\gamma_{3}\right| \leq \frac{1}{18}(3+4 \sqrt{2})=0.4809$ for functions $f$ in the class $\mathcal{K}_{0}$ without the assumption requiring the second coefficient of the corresponding starlike function $g(z)$ be real. However, this improved bound is still not sharp. Consequently, the problem of finding the sharp upper bound for $\left|\gamma_{3}\right|$ for the classes $\mathcal{K}_{0}$ as well as $\mathcal{K}$ is still open.

In the present paper we consider the following three familiar subclasses of closeto-convex functions

$$
\begin{aligned}
& \mathcal{F}_{1}:=\left\{f \in \mathcal{A}: \operatorname{Re}(1-z) f^{\prime}(z)>0 \quad \text { for } z \in \mathbb{D}\right\} \\
& \mathcal{F}_{2}:=\left\{f \in \mathcal{A}: \operatorname{Re}\left(1-z^{2}\right) f^{\prime}(z)>0 \text { for } z \in \mathbb{D}\right\} \\
& \mathcal{F}_{3}:=\left\{f \in \mathcal{A}: \operatorname{Re}\left(1-z+z^{2}\right) f^{\prime}(z)>0 \quad \text { for } z \in \mathbb{D}\right\} .
\end{aligned}
$$

The region of variability for the classes $\mathcal{F}_{1}, \mathcal{F}_{2}$ and $\mathcal{F}_{3}$ have been extensively studied by Ponnusamy, Vasudevarao and Yanagihara ([15], [16]). The main aim of this paper is to determine the sharp upper bounds for $\left|\gamma_{1}\right|,\left|\gamma_{2}\right|$ and $\left|\gamma_{3}\right|$ for functions $f$ in the classes $\mathcal{F}_{1}, \mathcal{F}_{2}$ and $\mathcal{F}_{3}$.

\section{Main Results}

Throughout the remainder of this paper, we assume that $f \in \mathcal{K}_{0}$ and $h \in \mathcal{P}$ have the series representations (1.1) and (1.2) respectively. Further, assume that $g \in \mathcal{S}^{*}$ has the following series representation:

$$
g(z)=z+\sum_{n=2}^{\infty} b_{n} z^{n} .
$$

It is not difficult to see that the function $H_{t, \mu}(z)$ given by

$$
H_{t, \mu}(z)=(1-2 t)\left(\frac{1+z}{1-z}\right)+t\left(\frac{1+\mu z}{1-\mu z}\right)+t\left(\frac{1+\bar{\mu} z}{1-\bar{\mu} z}\right)
$$

belongs to the class $\mathcal{P}$ for $0 \leq t \leq 1 / 2$ and $|\mu|=1$. Since $f \in \mathcal{K}_{0}$, there exists an $h \in \mathcal{P}$ such that

$$
z f^{\prime}(z)=g(z) h(z) .
$$

Using the representations (1.1), (1.2) and (2.1) in (2.2) we obtain

$$
z+\sum_{n=2}^{\infty} n a_{n} z^{n}=\left(z+\sum_{n=2}^{\infty} b_{n} z^{n}\right)\left(1+\sum_{n=1}^{\infty} c_{n} z^{n}\right) .
$$

Comparing the coefficients on both the sides of (2.3), we obtain

$$
\begin{aligned}
& 2 a_{2}=b_{2}+c_{1} \\
& 3 a_{3}=b_{3}+b_{2} c_{1}+c_{2} \\
& 4 a_{4}=b_{4}+c_{1} b_{3}+c_{2} b_{2}+c_{3} .
\end{aligned}
$$


A substitution of (2.4) in (1.7) gives

$$
\gamma_{1}=\frac{1}{4}\left(b_{2}+c_{1}\right) \text {. }
$$

An application of the triangle inequality to (2.7) gives

$$
4\left|\gamma_{1}\right| \leq\left|b_{2}\right|+\left|c_{1}\right| \text {. }
$$

Substituting (2.4) and (2.5) in (1.8), we obtain

$$
\gamma_{2}=\frac{1}{48}\left(8 b_{3}+2 b_{2} c_{1}+8 c_{2}-3 b_{2}^{2}-3 c_{1}^{2}\right) \text {. }
$$

Let $c_{1}=d e^{i \alpha}$ and $q=\cos \alpha$ with $0 \leq d \leq 2$ and $0 \leq \alpha<2 \pi$. Applying the triangle inequality in conjunction with Lemma 1.4 allows us to rewrite (2.9) as

$$
6\left|\gamma_{2}\right| \leq 2-\frac{d^{2}}{2}+\frac{1}{8}\left|\left(d q+b_{2}+i d \sqrt{1-q^{2}}\right)^{2}+\left(8 b_{3}-4 b_{2}^{2}\right)\right| .
$$

Substituting (2.4), (2.5) and (2.6) in (1.9), we obtain

$$
\gamma_{3}=\frac{1}{48}\left(6 c_{3}-b_{2}^{2} c_{1}-b_{2} c_{1}^{2}+2 b_{2} c_{2}+2 b_{3} c_{1}+b_{2}^{3}-4 b_{3} b_{2}+6 b_{4}+c_{1}^{3}-4 c_{1} c_{2}\right) \text {. }
$$

A simple application of Lemma 1.3 to (2.11) shows that

$$
\begin{gathered}
96 \gamma_{3}=6 t\left(1-|x|^{2}\right)\left(4-c_{1}^{2}\right)+c_{1}^{3}+\left(4 b_{3}-2 b_{1}^{2}\right) c_{1}+\left(2 b_{2}^{3}-8 b_{2} b_{3}+2 b_{4}\right) \\
+x\left(4-c_{1}^{2}\right)\left(2 b_{2}+2 c_{1}-3 c_{1} x\right) .
\end{gathered}
$$

Let $b_{n}$ be real for all $n \in \mathbb{N}$. Let $c_{1}=c$ and assume that $0 \leq c \leq 2$. Let $x=r e^{i \theta}$ and $p=\cos \theta$ with $0 \leq r \leq 1$ and $0 \leq \theta<2 \pi$. Taking modulus on both the sides of (2.12) and applying the triangle inequality we obtain

$$
96\left|\gamma_{3}\right| \leq 6\left(1-r^{2}\right)\left(4-c^{2}\right)+|\phi(c, r, p)|
$$

where

$$
\phi(c, r, p)=c^{3}+\left(4 b_{3}-2 b_{1}^{2}\right) c+\left(2 b_{2}^{3}-8 b_{2} b_{3}+2 b_{4}\right)+r e^{i \theta}\left(4-c^{2}\right)\left(2 b_{2}+2 c-3 c r e^{i \theta}\right) \text {. }
$$

Theorem 2.14. Let $f \in \mathcal{F}_{1}$ be given by (1.1). Then

(i) $\left|\gamma_{1}\right| \leq \frac{3}{4}$,

(ii) $\left|\gamma_{2}\right| \leq \frac{4}{9}$.

(iii) If $1 / 2 \leq a_{2} \leq 3 / 2$ then $\left|\gamma_{3}\right| \leq \frac{1}{288}(11+15 \sqrt{30})$.

The inequalities are sharp.

Proof. Let $f \in \mathcal{F}_{1}$. Then $f$ is a close-to-convex function with respect to the starlike function $g(z)=z /(1-z)$. In view of $(2.2)$ the function $f(z)$ can be written as

$$
z f^{\prime}(z)=\frac{z}{1-z} h(z) \text {. }
$$

As $\left|c_{1}\right| \leq 2$ for $h \in \mathcal{P}$ (see [9, Ch 7, Theorem 3]) a comparison of the R.H.S. of (2.2) and (2.15), shows that (2.8) reduces to

$$
4\left|\gamma_{1}\right| \leq 1+\left|c_{1}\right| \leq 3
$$


A function $p \in \mathcal{P}$ having $\left|c_{1}\right|=2$ is given by $p(z)=L_{1, \theta}(z)$ for $0 \leq \theta<2 \pi$ and substituting $p(z)$ in place of $h(z)$ in (2.15) determines a function $f \in \mathcal{F}_{1}$ for which the upper bound on $\left|\gamma_{1}\right|$ is sharp.

In view of (2.2) and (2.15), we can rewrite (2.10) as

$$
6\left|\gamma_{2}\right| \leq 2-\frac{\left|c_{1}\right|^{2}}{2}+\frac{1}{8} \sqrt{\left(d^{2}+5+2 d q\right)^{2}-16 d^{2}\left(1-q^{2}\right)}=: g(d, q)
$$

In view of (2.17) it suffices to find points in the square $S:=[0,2] \times[-1,1]$ where $g(d, q)$ attains the maximum value to determine the maximum value of $\left|\gamma_{2}\right|$. Solving $\frac{\partial g(d, q)}{\partial d}=0$ and $\frac{\partial g(d, q)}{\partial q}=0$ shows that there is no real valued solution to the pair of equations. Thus $g(d, q)$ does not attain maximum in the interior of $S$.

On the side $d=0, g(d, q)$ reduces to $g(0, q)=21 / 8$. On the side $d=2, g(d, q)$ can be written as $g(2, q)=\frac{1}{8} \sqrt{80 t^{2}+72 t+17}$. An elementary calculation shows that $\max _{-1 \leq q \leq 1} g(2, q)=g(2,1)=1.625$.

On the side $q=-1, g(d, q)$ maybe simplified to $g(d,-1)=\left(21-2 d-3 d^{2}\right) / 8$. It is not difficult to see that $g(d, 1)$ is decreasing for $c \in[0,2]$. Thus $\max _{0 \leq d \leq 2} g(d,-1)=$ $d(0,-1)=21 / 8=2.625$.

On the side $q=1, g(d, q)$ becomes $g(d, 1)=\left(21+2 d-3 d^{2}\right) / 8$. An elementary computation shows that $\max _{0 \leq d \leq 2} g(d, 1)=d(1 / 3,1)=8 / 3$.

Thus the maximum value of $g(d, q)$ and consequently that of $\left|\gamma_{2}\right|$ is attained at $(d, q)=(1 / 3,1)$, i.e., at $c_{1}=1 / 3$. Thus, from (2.17) we obtain $\left|\gamma_{2}\right| \leq 4 / 9$. Therefore in view of $(2.15)$ and Lemma 1.4 the equality holds in (ii) for the function $\widetilde{F}_{1} \in \mathcal{F}_{1}$ such that $z \widetilde{F_{1}^{\prime}}(z)=z(1-z)^{-1} L_{t, \theta}(z)$ with $t=1 / 6$ and $\theta=0$.

In view of (2.15), we may rewrite (2.13) as

$$
48\left|\gamma_{3}\right| \leq 3\left(4-c^{2}\right)\left(1-r^{2}\right)+\sqrt{\phi_{1}(c, r, p)},
$$

where

$$
\begin{aligned}
\phi_{1}(c, r, p)= & \left(\frac{c^{3}}{2}+c+3\right)^{2}+\left(4-c^{2}\right)^{2} r^{2}\left(-3 c^{2} p r+\frac{9}{4} c^{2} r^{2}+c^{2}-3 c p r+2 c+1\right) \\
& +2\left(\frac{c^{3}}{2}+c+3\right)\left(4-c^{2}\right) r\left(\frac{3}{2} c r-3 c p^{2} r-1+c p+p\right) .
\end{aligned}
$$

Let $G(c, r, p)=3\left(4-c^{2}\right)\left(1-r^{2}\right)+\sqrt{\phi_{1}(c, r, p)}$. Thus it suffices to find points in the closed cuboid $R:=[0,2] \times[0,1] \times[-1,1]$ where $G(c, r, p)$ attains the maximum value. We accomplish this by finding the maximum values in the interior of the six faces, on the twelve edges and in the interior of $R$.

On the face $\mathrm{c}=0$, it can be seen that $G(c, r, p)$ reduces to

$$
G(0, r, p)=\sqrt{24 p r+16 r^{2}+9}+12\left(1-r^{2}\right) .
$$

To determine the points on this face where the maxima occur, we solve $\frac{\partial G(0, r, p)}{\partial r}=0$ and $\frac{\partial G(0, r, p)}{\partial p}=0$. The only solution for this pair of equations is $(r, p)=(0,0)$. Thus, no maxima occur in the interior of the face $c=0$. 
On the face $\mathrm{c}=2, G(c, r, p)$ becomes $G(2, r, p)=9$ and hence

$$
\max _{0<r<1,-1<p<1} G(2, r, p)=9 .
$$

On the face $r=0, G(c, r, p)$ reduces to

$$
G(c, 0, p)=12-3 c^{2}+\frac{1}{2}\left(c^{3}+2 c+6\right) .
$$

To determine points where maxima occur, it suffices to find points where $\frac{\partial G(c, 0, p)}{\partial c}=0$ because $G(c, 0, p)$ is independent of $p$. The set of all such points is $\left\{\frac{1}{3}(6-\sqrt{30})\right\} \times$ $\{0\} \times[-1,1]$ and hence $G\left(\frac{1}{3}(6-\sqrt{30}), 0, p\right)=\frac{10 \sqrt{10}}{3 \sqrt{3}}+9=15.0858$. Thus

$$
\max _{0<c<2,-1<p<1} G(c, 0, p)=\frac{10 \sqrt{10}}{3 \sqrt{3}}+9=15.0858 .
$$

On the face $r=1, G(c, r, p)$ reduces to

$$
G(c, 1, p)=\sqrt{\psi_{1}(c, p)+\frac{1}{2}\left(c^{2}-4\right)\left(c^{3}+2 c+6\right)\left(6 c p^{2}-2 c p-2 p-3 c\right)}
$$

where

$$
\psi_{1}(c, p)=\left(\frac{c^{3}}{2}+c+3\right)^{2}+\left(c^{2}-4\right)^{2}\left(\frac{1}{4}\left(c^{2}-12 p c+8 c\right)+1\right) .
$$

A computation shows that $\frac{\partial G(c, 1, p)}{\partial p}=0$ yields

$$
p=\frac{2 c^{4}+2 c^{3}-5 c^{2}-2 c+3}{3 c\left(c^{3}+2 c+6\right)} .
$$

A more involved computation shows that $\frac{\partial G(c, 1, p)}{\partial c}=0$ implies

$$
\begin{aligned}
& \left(9 c^{5}-12 c^{3}+27 c^{2}-24 c-36\right) p^{2}-\left(12 c^{5}+10 c^{4}-52 c^{3}-30 c^{2}+46 c+8\right) p \\
& +\left(6 c^{5}+5 c^{4}-42 c^{3}-33 c^{2}+57 c+37\right)=0 .
\end{aligned} .
$$

Substituting (2.22) in (2.23) and performing a lengthy computation gives

$$
\frac{\left(c^{3}-7 c-3\right) \zeta_{1}(c)}{3 c^{2}\left(c^{3}+2 c+6\right)^{2}}=0
$$

where

$\zeta_{1}(c)=6 c^{10}-5 c^{9}+20 c^{8}+86 c^{7}-49 c^{6}+257 c^{5}+623 c^{4}-629 c^{3}-1095 c^{2}-60 c+36$.

The numerical solutions of (2.24) such that $0<c<2$ are $c \approx 0.151355$ and $c \approx 1.30718$. Substituting these values of $c$ in $(2.22)$ gives $p \approx 0.904769$ and $p \approx 0.050509$. The corresponding values of $G(c, 1, p)$ are $G(0.151355,1,0.904769)=$ 6.83676 and $G(1.30718,1,0.050509)=11.2488$ respectively.

As $G(c, 1, p)$ is uniformly continuous on $[0,2] \times\{1\} \times[-1,1]$, the difference between extremum values of $G(c, 1, p)$ and either of 6.83676 or 11.2488 can be made smaller than an $\epsilon \ll 1$. Therefore

$$
\max _{0<c<2,-1<p<1} G(c, 1, p) \approx 11.2488 .
$$


On the face $p=-1, G(c, r, p)$ reduces to

$G(c, r,-1)=\frac{1}{2}\left(3 r^{2}+2 r+1\right) c^{3}+\left(3 r^{2}+r-3\right) c^{2}-\left(6 r^{2}+4 r-1\right) c-\left(12 r^{2}+4 r-15\right)$.

Now we show that $\frac{\partial G(c, r,-1)}{\partial c}=0$ and $\frac{\partial G(c, r,-1)}{\partial r}=0$ have no solution in the interior of this face. On the contrary, assume that $\frac{\partial G(c, r,-1)}{\partial c}=0$ and $\frac{\partial G(c, r,-1)}{\partial r}=0$ have a solution in the interior of the face $p=-1$. Then $\frac{\partial G(c, r,-1)}{\partial r}=0$ gives

$$
r=\frac{c+1}{3(2-c)}
$$

By substituting (2.26) in $\frac{\partial G(c, r,-1)}{\partial c}=0$, we obtain $c=\frac{1}{6}(-4 \pm \sqrt{190})$, both of which lie outside the range of $c \in[0,2]$.

On the face $p=1, G(c, r, p)$ reduces to

$G(c, r, 1)=\frac{1}{2}\left(3 r^{2}-2 r+1\right) c^{3}+\left(3 r^{2}-r-3\right) c^{2}-\left(6 r^{2}+4 r-1\right) c-\left(12 r^{2}-4 r-15\right)$.

At the points where $G(c, r, 1)$ attains the maximum value, $\frac{\partial G(c, r, 1)}{\partial c}$ and $\frac{\partial G(c, r, 1)}{\partial r}$ necessarily vanish. The solution to the pair of equations $\frac{\partial G(c, r, 1)}{\partial c}=0$ and $\frac{\partial G(c, r, 1)}{\partial r}=0$ is $(c, r)=\left(\frac{1}{2}(60-\sqrt{30}), \frac{1}{105}(25-\sqrt{30})\right)$ and subsequently

$$
G\left(\frac{1}{2}(6-\sqrt{30}), \frac{1}{105}(25-\sqrt{30}), 1\right)=5 \sqrt{\frac{15}{2}}+\frac{11}{6}=15.5264 .
$$

Further computations show that

$$
\max _{0<c<2,0<r<1} G(c, r, 1)=\sqrt{\frac{15}{2}}+\frac{11}{6}=15.5264 .
$$

Now we find out the maximum values attained by $G(c, r, p)$ on the edges of $R$. Evaluating (2.19) ) on the edge $c=0, p=1$ we obtain $G(0, r, 1)=12\left(1-r^{2}\right)+4 r+3$. A simple computation shows that the maximum of $G(0, r, 1)$ is $46 / 3$ which occurs at $r=1 / 6$. At the end points of this edge, we have $G(0,0,1)=15$ and $G(0,1,1)=7$. Hence

$$
\max _{0 \leq r \leq 1} G(0, r, 1)=\frac{46}{3} \text {. }
$$

In view of (2.19), we obtain by a series of straightforward computations the maximum value of $G(c, r, p)$ on the edges $c=0, r=0 ; c=0, r=1$ and $c=0, p=-1$ as

$$
\max _{-1 \leq p \leq 1} G(0,0, p)=15, \quad \max _{-1 \leq p \leq 1} G(0,1, p)=7 \quad \text { and } \quad \max _{0 \leq r \leq 1} G(0, r,-1)=15 .
$$

A simple observation shows that $G(2, r, p)=9$ implies

$$
\max _{-1 \leq p \leq 1} G(2,0, p)=\max _{-1 \leq p \leq 1} G(2,1, p)=\max _{0 \leq r \leq 1} G(2, r,-1)=\max _{0 \leq r \leq 1} G(2, r, 1)=9 .
$$

As (2.20) is independent of $p$, the maximum value of $G(c, r, p)$ on the edges $r=$ $0, p=-1$ and $r=0, p=1$ is

$$
\max _{0 \leq c \leq 2} G(c, 0,-1)=\max _{0 \leq c \leq 2} G(c, 0,1)=15.0858 .
$$


On the edge $r=1, p=-1$, (2.21) can be simplified to $G(c, 1,-1)=\left|3 c^{3}+c^{2}-9 c-1\right|$. A straightforward calculation shows that

$$
\max _{0 \leq c \leq 2} G(c, 1,-1)=9 .
$$

On the edge $r=1, p=1$, (2.21) reduces to $G(c, 1,1)=c^{3}-c^{2}-c+7$. A simple computation shows that

$$
\max _{0 \leq c \leq 2} G(c, 1,1)=9 .
$$

Now we show that $G(c, r, p)$ does not attain maximum value in the interior of the cuboid $R$. In order to find the points where the maximum value is obtained in the interior of $R$, we solve $\frac{\partial G(c, r, p)}{\partial c}=0, \frac{\partial G(c, r, p)}{\partial r}=0$ and $\frac{\partial G(c, r, p)}{\partial p}=0$. A computation shows that $\frac{\partial G(c, r, p)}{\partial p}=0$ implies

$$
p=\frac{3 c^{4} r^{2}+c^{4}+3 c^{3} r^{2}+c^{3}-12 c^{2} r^{2}+2 c^{2}-12 c r^{2}+8 c+6}{6 c\left(c^{3}+2 c+6\right) r} .
$$

By substituting (2.27) in $\frac{\partial G(c, r, p)}{\partial r}=0$, we get

$$
r=\frac{\sqrt{c^{3}+2 c+6}}{\sqrt{3} \sqrt{c^{3}-4 c}}
$$

It is easy to see that $\frac{c^{3}+2 c+6}{3\left(c^{3}-4 c\right)}$ is negative for all values of $c \in[0,2]$. Hence there cannot be an extremum inside the cuboid $R$. This shows that the maximum value of $\left|\gamma_{3}\right|$ is $\frac{1}{48}\left(5 \sqrt{\frac{15}{2}}+\frac{11}{6}\right)$ for $(c, r, p)=\left(\frac{1}{2}(6-\sqrt{30}), \frac{1}{105}(25-\sqrt{30}), 1\right)$.

Let $c=c_{1}$ and $(c, r, p)=\left(\frac{1}{2}(6-\sqrt{30}), \frac{1}{105}(25-\sqrt{30}), 1\right)$. Then in view of Lemma 1.3 we obtain $c_{2}=\frac{1}{12}(76-13 \sqrt{30})$ and $c_{3}=\frac{1}{72}(554-75 \sqrt{30})$. It is not difficult to see that a function $G^{*} \in \mathcal{P}$ having

$$
\left(c_{1}, c_{2}, c_{3}\right)=\left(\frac{1}{2}(6-\sqrt{30}), \frac{1}{12}(76-13 \sqrt{30}), \frac{1}{72}(554-75 \sqrt{30})\right)
$$

is given by $G^{*}(z)=H_{t_{1}, \mu_{1}}(z)$ where $\mu_{1}=\frac{1}{12}(-1-\sqrt{30})+i \frac{1}{12} \sqrt{113-2 \sqrt{30}}$, and $t_{1}=\frac{3}{278}(15 \sqrt{30}-56)$. Therefore the bound in (iii) is sharp for the function $F_{1}(z)$ such that

$$
z F_{1}^{\prime}(z)=\frac{z}{1-z} G^{*}(z)
$$

Theorem 2.29. Let $f \in \mathcal{F}_{2}$ be given by (1.1). Then

(i) $\left|\gamma_{1}\right| \leq \frac{1}{4}$

(ii) $\left|\gamma_{2}\right| \leq \frac{1}{2}$.

(iii) If $0 \leq a_{2} \leq 1$ then $\left|\gamma_{3}\right| \leq \frac{1}{972}(95+23 \sqrt{46})$.

The inequalities are sharp.

Proof. Let $f \in \mathcal{F}_{2}$. It is evident that $f$ is close-to-convex with respect to the starlike function $g(z)=z /\left(1-z^{2}\right)$. From (2.2), $f(z)$ can be written as

$$
z f^{\prime}(z)=\frac{z}{1-z^{2}} h(z) .
$$


Thus in view of (2.30), (2.8) reduces to

$$
4\left|\gamma_{1}\right| \leq\left|c_{1}\right|
$$

Noting that $\left|c_{1}\right| \leq 2$, (2.31) then implies that $\left|\gamma_{1}\right| \leq 1 / 2$. It is easy to see that A function $p \in \mathcal{P}$ having $\left|c_{1}\right|=2$ is given by $p(z)=L_{1, \theta}(z)$ for $0 \leq \theta<2 \pi$. Substituting $L_{1, \theta}(z)$ in place of $h(z)$ in (2.30) shows that (i) is sharp.

A comparison of (2.30) and (2.2) shows that (2.9) reduces to

$$
6 \gamma_{2} \leq\left(c_{2}-\frac{3}{8} c_{1}^{2}\right)+1
$$

Applying the triangle inequality in conjunction with Lemma 1.5 with $\mu=3 / 8$ shows that $\left|\gamma_{2}\right| \leq 1 / 2$. It is evident from Lemma 1.5 that the equality holds in (ii) for the function $\widetilde{F_{2}}(z)$ such that $z{\widetilde{F_{2}}}^{\prime}(z)=z\left(1-z^{2}\right)^{2} L_{0,0}(z)$.

Considering (2.30) as an instance of (2.2), (2.13) can be simplified to

$$
96\left|\gamma_{3}\right| \leq 6\left(4-c^{2}\right)\left(1-r^{2}\right)+c \sqrt{\phi_{2}(c, r, p)},
$$

where

$$
\begin{gathered}
\phi_{2}(c, r, p)=\left(c^{2}+4\right)^{2}+2 r\left(4-c^{2}\right)\left(4+c^{2}\right)\left(2 p+3 r-6 p^{2} r\right) \\
+r^{2}\left(4-c^{2}\right)^{2}\left(4+9 r^{2}-12 r p\right) .
\end{gathered}
$$

Let $F(c, r, p)=6\left(1-r^{2}\right)\left(4-c^{2}\right)+c \sqrt{\phi_{2}(c, r, p)}$. We find points where $F(c, r, p)$ attains the maximum value by finding its local maxima on the six faces and in the interior of $R$. On the face $c=0, F(c, r, p)$ becomes

$$
F(0, r, p)=24\left(1-r^{2}\right) \text {. }
$$

As $F(0, r, p)$ is a decreasing function of $r$, the maximum value of $F(0, r, p)$ is attained on the edge $c=0, r=0$. Consequently, we have

$$
\max _{0 \leq r \leq 1,-1 \leq p \leq 1} F(0, r, p)=24 .
$$

On the face $c=2, F(c, r, p)$ becomes $F(2, r, p)=16$ and hence

$$
\max _{0 \leq r \leq 1,-1 \leq p \leq 1} F(2, r, p)=16 .
$$

On the face $r=0$, we can simplify $F(c, r, p)$ as

$$
F(c, 0, p)=24-6 c^{2}+c\left(c^{2}+4\right) .
$$

Since $F(c, 0, p)$ is independent of $p$, we find the set of all points where $\frac{\partial F(c, 0, p)}{\partial c}$ vanishes as $\left\{\frac{2}{3}(3-\sqrt{6})\right\} \times\{0\} \times[-1,1]$ and hence $F\left(\frac{2}{3}(3-\sqrt{6}), 0, p\right)=\frac{16}{9}(9+2 \sqrt{6})=$ 24.7093. Evaluating (2.34) on the edges $c=0, r=0$ and $c=2, r=0$, we obtain

$$
\max _{0 \leq c \leq 2,-1 \leq p \leq 1} F(c, 0, p)=24.7093 .
$$

On the face $r=1, F(c, r, p)$ reduces to

$$
F(c, 1, p)=2 c \sqrt{24 c^{2}(p-1)-16(p-1)(5+3 p)+c^{4}\left(2-4 p+3 p^{2}\right)} .
$$


We solve $\frac{\partial F(c, 1, p)}{\partial c}=0$ and $\frac{\partial F(c, 1, p)}{\partial p}=0$ to determine points where maxima occur in the face $r=1$. A computation shows that $\frac{\partial F(c, 1, p)}{\partial p}=0$ implies

$$
p=\frac{2\left(c^{2}-2\right)}{3\left(c^{2}+4\right)} .
$$

A slightly involved computation shows that $\frac{\partial F(c, 1, p)}{\partial c}=0$ gives

$$
\left(18 c^{4}-96\right) p^{2}-8\left(3 c^{4}-12 c^{2}+8\right) p+\left(12 c^{4}-96 c^{2}+160\right)=0 .
$$

Substituting (2.36) in (2.37) followed by a computation gives

$$
\frac{4\left(3 c^{8}-160 c^{4}-512 c^{2}+2048\right)}{3\left(c^{2}+4\right)^{2}}=0 .
$$

The numerical solution of (2.38) in $0<c<2$ is $c \approx 1.54836$. Using (2.36) we then obtain $p \approx 0.414152$. Therefore $F(1.54836,1,0.414152)=18.0595$.

Using uniform continuity of $F(c, 1, p)$ on $[0,2] \times\{1\} \times[-1,1]$ we infer that the difference between the maximum value of $F(c, 1, p)$ and 18.0595 can be made smaller than an $\epsilon \ll 1$. On the edge $c=0, r=1, F(c, r, p)$ becomes $F(0,1, p)=0$. On the edge $c=2, r=1, F(c, r, p)$ becomes $F(2,1, p)=16$. On the edge $r=1, p=-1$, (2.35) can be simplified to $F(c, 1,-1)=2 c\left|3 c^{2}-8\right|$. It is easy to see that $F(c, 1,-1)$ has the maximum value 16 on $[0,2]$.

A simple computation shows that the maximum value of $F(c, r, p)$ on the edge $r=1, p=1$ is 16 . Therefore,

$$
\max _{0 \leq c \leq 2,-1 \leq p \leq 1} F(c, 1, p) \approx 18.0595 .
$$

On the face $p=-1, F(c, r, p)$ reduces to

$$
F(c, r,-1)=6\left(4-c^{2}\right)\left(1-r^{2}\right)+c\left|c^{2}+4-\left(2 r-3 r^{2}\right)\left(4-c^{2}\right)\right| .
$$

A computation similar to the one on the face $p=-1$ in Theorem 2.14 shows that $\frac{\partial F(c, r,-1)}{\partial c}=0$ and $\frac{\partial F(c, r,-1)}{\partial r}=0$ have no solution in the interior of the face $p=-1$. Thus the maximum value is attained on the edges.

On the edge $c=0, p=-1, F(c, r, p)$ becomes $F(0, r,-1)=24\left(1-r^{2}\right)$. The maximum value of $F(0, r,-1)$ is clearly 24 . On the edge $r=0, p=-1, F(c, r, p)$ becomes

$$
F(c, 0,-1)=6\left(4-c^{2}\right)+c\left(4+c^{2}\right) .
$$

The maximum value of $F(c, 0,-1)$ is $\frac{16}{9}(9+2 \sqrt{6})=24.7093$ (see the face $r=0$ ). The maximum values of $F(c, r, p)$ on the edges $c=2, p=-1$ and $r=1, p=-1$ are 16 and 10.0566 respectively (see the faces $c=2$ and $r=1$ ). Therefore

$$
\max _{0 \leq c \leq 2,0 \leq r \leq 1} F(c, r,-1)=\frac{16}{9}(9+2 \sqrt{6})=24.7093 .
$$

On the face $p=1, F(c, r, p)$ reduces to

$$
F(c, r, 1)=6\left(4-c^{2}\right)\left(1-r^{2}\right)+c\left|c^{2}+4+\left(2 r+3 r^{2}\right)\left(4-c^{2}\right)\right| .
$$

Solving $\frac{\partial F(c, r, 1)}{\partial c}=0$ and $\frac{\partial F(c, r, 1)}{\partial r}=0$ we obtain $(c, r)=\left(\frac{1}{3}(8-\sqrt{46}), \frac{1}{75}(11-\sqrt{46})\right)$ and hence $F\left(\frac{1}{3}(8-\sqrt{46}), \frac{1}{75}(11-\sqrt{46}), 1\right)=\frac{8}{81}(95+23 \sqrt{46})=24.7895$. It is 
not difficult to see that the maximum value of $F(c, r, 1)$ on the edges is 24.7093, which occurs on the edge $r=0, p=1$ (see the face $r=0$ ) as the computations for the edges have been done on earlier faces. Therefore

$$
\max _{0 \leq c \leq 2,0 \leq r \leq 1} F(c, r, 1)=\frac{8}{81}(95+23 \sqrt{46})=24.7895 .
$$

We now show that $F(c, r, p)$ cannot attain a maximum in the interior of the cuboid $R$. To determine points in the interior of $R$ where the maxima occurs (if any), we solve $\frac{\partial F(c, r, p)}{\partial c}=0, \frac{\partial F(c, r, p)}{\partial r}=0$ and $\frac{\partial F(c, r, p)}{\partial p}=0$. A computation shows that $\frac{\partial F(c, r, p)}{\partial p}=0$ implies

$$
p=\frac{3 c^{2} r^{2}+c^{2}-12 r^{2}+4}{6\left(c^{2}+4\right) r} .
$$

Using (2.39) in $\frac{\partial F(c, r, p)}{\partial r}=0$ and then solving for $r$ yields

$$
r=\frac{\sqrt{c^{2}+4}}{\sqrt{3} \sqrt{c^{2}-4}} .
$$

As $\frac{c^{2}+4}{3\left(c^{2}-4\right)}$ is negative for all values of $c \in[0,2]$, there cannot be an extremum in the interior of $R$. This proves that the maximum value of $\left|\gamma_{3}\right|$ is $\frac{1}{972}(95+23 \sqrt{46})$ for $(c, r, p)=\left(\frac{1}{3}(8-\sqrt{46}), \frac{1}{75}(11-\sqrt{46}), 1\right)$.

Let $c=c_{1}$ and $(c, r, p)=\left(\frac{1}{3}(8-\sqrt{46}), \frac{1}{75}(11-\sqrt{46}), 1\right)$. Then in view of Lemma 1.3, we obtain $c_{2}=\frac{1}{27}(134-19 \sqrt{46})$ and $c_{3}=\frac{2}{243}(721-71 \sqrt{46})$. It is not difficult to see that a function $F^{*} \in \mathcal{P}$ having

$$
\left(c_{1}, c_{2}, c_{3}\right)=\left(\frac{1}{3}(8-\sqrt{46}), \frac{1}{27}(134-19 \sqrt{46}), \frac{2}{243}(721-71 \sqrt{46})\right)
$$

is given by $F^{*}(z)=H_{t_{2}, \mu_{2}}(z)$ where $\mu_{2}=\frac{1}{18}(-1-\sqrt{46})+i \frac{1}{18} \sqrt{277-2 \sqrt{46}}$ and $t_{2}=$ $\frac{1}{10}(\sqrt{46}-4)$. This shows that the bound in (iii) is sharp for the function $F_{2}(z)$ such that $z F_{2}^{\prime}(z)=z\left(1-z^{2}\right)^{-1} F^{*}(z)$.

Theorem 2.40. Let $f \in \mathcal{F}_{3}$ be given by (1.1). Then
(i) $\left|\gamma_{1}\right| \leq \frac{3}{4}$,
(ii) $\left|\gamma_{2}\right| \leq \frac{2}{5}$.
(iii) If $1 / 2 \leq a_{2} \leq 3 / 2$ then $\left|\gamma_{3}\right| \leq \frac{743+131 \sqrt{262}}{7776}$.

The inequalities are sharp.

Proof. Let $f \in \mathcal{F}_{3}$. Then $f$ is close-to-convex with respect to the starlike function $g(z)=z /\left(1-z+z^{2}\right)$. In view of (2.2), $f(z)$ can be written as

$$
z f^{\prime}(z)=\frac{z}{1-z+z^{2}} h(z) .
$$

Therefore (2.8) reduces to

$$
4\left|\gamma_{1}\right| \leq 1+\left|c_{1}\right|
$$


Thus from (2.42) we obtain $\left|\gamma_{1}\right| \leq 3 / 4$ as $\left|c_{1}\right| \leq 2$ for $h \in \mathcal{P}$. A function in $\mathcal{P}$ having $\left|c_{1}\right|=2$ is given by $L_{1, \theta}(z), 0 \leq \theta<2 \pi$ The equality in (i) is attained for a function $\widetilde{f}(z)$ such that $z \widetilde{f}^{\prime}(z)=z\left(1-z+z^{2}\right)^{-1} L_{1, \theta}(z)$.

In view of (2.41), (2.10) becomes

$$
6\left|\gamma_{2}\right| \leq 2-\frac{\left|c_{1}\right|^{2}}{2}+\frac{1}{8} \sqrt{\left(d^{2}+1-2 d t\right)\left(d^{2}+9+6 d t\right)}=: k(d, q) .
$$

It is evident from (2.43) that it is sufficient to find the maximum value of $k(d, q)$ in the square $S$ to obtain the same for $\left|\gamma_{2}\right|$.

To obtain points where $k(d, q)$ attains maximum, we solve $\frac{\partial k(d, q)}{\partial d}=0$ and $\frac{\partial k(d, q)}{\partial q}=$ 0 . The solutions obtained are complex, showing that $k(d, q)$ does not attain maximum in the interior of $S$.

On the side $d=0, k(d, q)$ reduces to $k(d, q)=2.375$. On the side $d=2$, we see that $k(d, q)=(\sqrt{65+8 t-48 t}) / 8$. An elementary computation shows that $\max _{-1 \leq q \leq 1} k(2, q)=k(2,1 / 12)=1.01036$.

On the side $q=-1, k(d, q)$ becomes $k(d,-1)=\left(19+2 d-5 d^{2}\right) / 8$. A straightforward computation shows that $\max _{0 \leq d \leq 2} k(d,-1)=k(1 / 5,-1)=12 / 5=2.4$.

On the side $q=1, k(d, q)$ may be simplified as $k(d, 1)=\left(19-2 d-5 d^{2}\right) / 8$. As $k(d, 1)$ is a decreasing function for $d \in[0,2]$, we see that $\max _{0 \leq d \leq 2} k(d, 1)=k(0,1)=$ $19 / 8=2.375$.

Thus the maximum value of $k(d, q)$ in $S$ is $12 / 5$ and occurs at $(d, q)=(1 / 5,-1)$. Consequently, (2.43) implies that $\left|\gamma_{2}\right| \leq 2 / 5$, with the equality occurring for $c_{1}=$ $-1 / 5$.

Therefore, in view of Lemma 1.4, the equality in (ii) holds for the function $\widetilde{F_{2}}(z)$ such that $z \widetilde{F_{2}^{\prime}}(z)=z\left(1-z+z^{2}\right)^{-1} L_{t, \theta}(z)$ where $t=1 / 10$ and $\theta=\pi$.

Using (2.41) we may rewrite (2.13) as

$$
96\left|\gamma_{3}\right| \leq 6\left(1-r^{2}\right)\left(4-c^{2}\right)+\sqrt{\phi_{3}(c, r, p)}
$$

where

$$
\begin{gathered}
\phi_{3}(c, r, p)=\left(c^{3}-2 c-10\right)^{2}+2 r\left(4-c^{2}\right)\left(c^{3}-2 c-10\right)\left(2 p+2 c p-6 c r p^{2}+3 r c\right) \\
+r^{2}\left(4-c^{2}\right)^{2}\left(4 c^{2}+4+9 c^{2} r^{2}+8 c-12 c^{2} r p-12 c r p\right) .
\end{gathered}
$$

Let $K(c, r, p)=6\left(1-r^{2}\right)\left(4-c^{2}\right)+\sqrt{\phi_{3}(c, r, p)}$. We find the points in the cuboid $R$ where the maxima of $K(c, r, p)$ occur.

On the face $c=0, K(c, r, p)$ takes the following form

$$
K(0, r, p)=24\left(1-r^{2}\right)+2 \sqrt{25-40 r p+16 r^{2}} .
$$

By solving $\frac{\partial K(0, r, p)}{\partial r}=0$ and $\frac{\partial K(0, r, p)}{\partial p}=0$ we obtain $(r, p)=(0,0)$. Thus $K(c, r, p)$ does not attain maximum in the interior of the face $c=0$.

On the face $c=2, K(c, r, p)$ reduces to $K(2, r, p)=6$ and hence

$$
\max _{0<r<1,-1<p<1} K(2, r, p)=6 .
$$


On the face $r=0, K(c, r, p)$ may be simplified as

$$
K(c, 0, p)=6\left(4-c^{2}\right)+\left|c^{3}-2 c-10\right| .
$$

Since $K(c, 0, p)$ is independent of $p$, it suffices to find out points such that $\frac{\partial K(c, 0, p)}{\partial c}=$ 0 . The set of all such points is $\left\{\frac{1}{3}(-6+\sqrt{42})\right\} \times\{0\} \times[-1,1]$ and

$$
K\left(\frac{1}{3}(-6+\sqrt{42}), 0, p\right)=\frac{14}{9}(9+2 \sqrt{42})=34.1623 .
$$

Therefore

$$
\max _{0<c<2,-1<p<1} K(c, 0, p)=\frac{14}{9}(9+2 \sqrt{42})=34.1623 .
$$

On the face $r=1, K(c, r, p)$ becomes

$$
K(c, 1, p)=\sqrt{\psi_{3}(c, p)+2\left(c^{3}-2 c-10\right)\left(c^{2}-4\right)\left(6 c p^{2}-2 c p-2 p-3 c\right)}
$$

where

$$
\psi_{3}(c, p)=\left(c^{3}-2 c-10\right)^{2}+\left(c^{2}-4\right)^{2}\left(13 c^{2}-12 c^{2} p+8 c-12 c p+4\right) .
$$

A computation shows that $\frac{\partial K(c, 1, p)}{\partial p}=0$ implies

$$
p=\frac{2 c^{4}+2 c^{3}-7 c^{2}-12 c-5}{3 c\left(c^{3}-2 c-10\right)} .
$$

A lengthy computation shows that $\frac{\partial K(c, 1, p)}{\partial c}$ implies

$$
\begin{aligned}
& \left(9 c^{5}-36 c^{3}-45 c^{2}+24 c+60\right) p^{2}-\left(12 c^{5}+10 c^{4}-60 c^{3}-60 c^{2}+46 c+48\right) p \\
& +\left(6 c^{5}+5 c^{4}-34 c^{3}-9 c^{2}+33 c-9\right)=0
\end{aligned}
$$

Substituting (2.48) in (2.49) and then performing another lengthy computation gives

$$
\frac{\left(c^{3}-5 c+5\right) \zeta_{2}(c)}{3 c^{2}\left(c^{3}-2 c-10\right)^{2}}=0
$$

where

$\zeta_{2}(c)=6 c^{10}-5 c^{9}-32 c^{8}-104 c^{7}+147 c^{6}+375 c^{5}+459 c^{4}-375 c^{3}-1135 c^{2}+140 c+100$.

The numerical solutions of (2.50) are obtained as $c \approx 0.354278$ and $c \approx 1.27688$. Further computations show that $K(c, 1, p)$ does not attain a maxima at these points even though the partial derivatives vanish. On the face $p=-1, K(c, r, p)$ reduces to

$$
K(c, r,-1)=6\left(1-r^{2}\right)\left(4-c^{2}\right)-\left(c^{3}-2 c-10\right)+2\left(4-c^{2}\right)\left(2+2 c+3 c r^{2}\right) .
$$

By solving $\frac{\partial K(c, r,-1)}{\partial c}=0$ and $\frac{\partial K(c, r,-1)}{\partial r}=0$ we obtain $c=\frac{1}{6}(-14+\sqrt{262})$ and $r=\frac{1}{69}(3+\sqrt{262})$. The corresponding maximum value is

$$
K\left(\frac{1}{6}(-14+\sqrt{262}), \frac{1}{69}(3+\sqrt{262}),-1\right)=\frac{1}{81}(743+131 \sqrt{262})=35.3509 .
$$

Therefore

$$
\max _{0<c<2,0<r<1} K(c, r,-1)=35.3509
$$


On the face $p=1, K(c, r, p)$ reduces to

$$
K(c, r, 1)=6\left(1-r^{2}\right)\left(4-c^{2}\right)-\left(c^{3}-2 c-10\right)+\left(4-c^{2}\right)\left(3 c r^{2}-2-2 c\right) .
$$

It is not difficult to see that $\frac{\partial K(c, r, 1)}{\partial c}=0$ and $\frac{\partial K(c, r, 1)}{\partial r}=0$ have no solution in the interior of the face $p=1$. Thus $K(c, r, p)$ does not attain maximum in the interior of this face.

Now we find the maximum values attained on the edges of $\mathrm{R}$. It is evident from (2.45) that on the edges $c=0, r=0$ and $c=0, r=1$, the maximum values of $K(c, r, p)$ are

$$
\max _{-1 \leq p \leq 1} K(0,0, p)=34 \quad \text { and } \quad \max _{-1 \leq p \leq 1} K(0,1,-1)=18 .
$$

On the edge $c=0, p=-1$, (2.45) reduces to $K(0, r,-1)=24\left(1-r^{2}\right)+2(5+4 r)$. An elementary computation shows that the maximum value of $K(0, r,-1)$ is attained at $\left(0, \frac{1}{6},-1\right)$ and $\max _{0 \leq r \leq 1} K(0, r,-1)=104 / 3$.

On the edge $c=0, p=1$, (2.45) reduces to $K(0, r, 1)=24\left(1-r^{2}\right)+2(5-4 r)$. A computation shows that $\max _{0 \leq r \leq 1} K(0, r, 1)=34$.

It is evident that $K(2, r, p)=6$ implies

$$
\max _{-1 \leq p \leq 1} K(2,0, p)=\max _{-1 \leq p \leq 1} K(2,1, p)=\max _{0 \leq r \leq 1} K(2, r,-1)=\max _{0 \leq r \leq 1} K(2, r, 1)=6 .
$$

Considering (2.46) and the maximum value of $K(c, 0, p)$ we obtain the maximum values on the edges $r=0, p=-1$ and $r=0, p=1$ as

$$
\max _{0 \leq c \leq 2} K(c, 0,-1)=\max _{0 \leq c \leq 2} K(c, 0,1)=34.1623 .
$$

On the edge $r=1, p=-1$, (2.47) maybe be simplified as

$$
\begin{aligned}
& K(c, 1,-1) \\
= & \sqrt{\left(c^{3}-2 c-10\right)^{2}-2(5 c+2)\left(c^{3}-2 c-10\right)\left(4-c^{2}\right)+\left(25 c^{2}+20 c+4\right)\left(4-c^{2}\right)^{2}} .
\end{aligned}
$$

A computation shows that $K(c, 1,-1)$ attains the local maximum at $(1,1,-1)$ and $\max _{0 \leq c \leq 2} K(c, 1,-1)=32$.

On the edge $r=1, p=1$, (2.47) reduces to $K(c, 1,1)=2\left(1+3 c+c^{2}-c^{3}\right)$. An elementary computation shows that

$$
\max _{0 \leq c \leq 2} K(c, 1,1)=K\left(\frac{1}{3}(1+\sqrt{10}), 1,1\right)=\frac{8}{27}(14+5 \sqrt{10})=8.833 .
$$

Now we show that $K(c, r, p)$ does not attain maximum in the interior of the cuboid $R$. At the points where the maxima occur in the cuboid $R$ we have $\frac{\partial K(c, r, p)}{\partial c}=$ $0, \frac{\partial K(c, r, p)}{\partial r}=0$ and $\frac{\partial K(c, r, p)}{\partial p}=0$. A computation shows that $\frac{\partial K(c, r, p)}{\partial p}=0$ implies

$$
p=\frac{3 c^{4} r^{2}+c^{4}+3 c^{3} r^{2}+c^{3}-12 c^{2} r^{2}-2 c^{2}-12 c r^{2}-12 c-10}{6 c\left(c^{3}-2 c-10\right) r} .
$$


Substituting (2.52) in $\frac{\partial K(c, r, p)}{\partial r}=0$ and then solving for $r$ we obtain

$$
r=\frac{\sqrt{c^{3}-2 c-10}}{\sqrt{3 c^{3}-12 c}} .
$$

Substituting (2.53) in (2.52) gives

$$
p=\frac{(c+1) \sqrt{c\left(c^{2}-4\right)}}{\sqrt{3} c \sqrt{c^{3}-2 c-10}}
$$

Substituting (2.53) and (2.54) in $\frac{\partial K(c, r, p)}{\partial c}=0$, we obtain

$$
\frac{8\left(c^{3}-5 c+5\right)}{c^{2}-4}=0 .
$$

It can be seen that the roots to the above equation are either negative or imaginary. This shows that a maximum cannot be attained inside $R$. Thus we see that the maximum value for $\left|\gamma_{3}\right|$ is attained for

$$
(c, r, p)=\left(\frac{1}{6}(-14+\sqrt{262}), \frac{1}{69}(3+\sqrt{262}),-1\right)
$$

and is equal to $(743+131 \sqrt{262}) / 81=35.3509$. Using these values of $(c, r, p)$ in Lemma 1.3, we obtain $c_{2}=\frac{1}{108}(548-37 \sqrt{262})$ and $c_{3}=\frac{47525 \sqrt{262}-698926}{44712}$. Therefore for given

$$
\left(c_{1}, c_{2}, c_{3}\right)=\left(\frac{1}{6}(-14+\sqrt{262}), \frac{1}{108}(548-37 \sqrt{262}), \frac{47525 \sqrt{262}-698926}{44712}\right)
$$

there exists a function $K^{*} \in \mathcal{P}$ given by $K^{*}(z)=H_{t_{3}, \mu_{3}}(z)$, where

$$
\mu_{3}=\frac{-769+35 \sqrt{262}}{828}+i \frac{\sqrt{-226727+53830 \sqrt{262}}}{828} \quad \text { and } t_{3}=\frac{32352-687 \sqrt{262}}{64622} \text {. }
$$

The inequality (iii) is sharp for the function $F_{3}(z)$ such that

$$
z F_{3}^{\prime}(z)=\frac{z}{1-z+z^{2}} K^{*}(z) \text {. }
$$

\section{REFERENCES}

[1] A. Bielecki and Z. Lewandowski, Sur un théorème concernant les fonctions univalentes linéairement accessibles, Annales Polonici Mathematici. Vol. 1. (12), (1962) 61-63.

[2] M. Biernacki, Sur la représentation conforme des domaines linéairement accessibles. Prace Matematyczno-Fizyczne. 44 (1937), 293-314.

[3] L. De Branges, A proof of the Bieberbach conjecture. Acta Math. 154 (1985), no. 1-2, $137-152$.

[4] P. L. DuREn, Univalent functions (Grundlehren der mathematischen Wissenschaften 259, New York, Berlin, Heidelberg, Tokyo), Springer-Verlag, 1983.

[5] P. L. Duren and Y. J. Leung, Logarithmic coefficients of univalent functions, J. Analyse Math. 36 (1979), 36-43.

[6] M. M. ElHosh, On the logarithmic coefficients of close-to-convex functions, J. Austral. Math. Soc. Ser. A 60 (1996), 1-6. 
[7] Md. Firoz Ali and A. Vasudevarao, On logarithmic coefficients of some close-to-convex functions, arXiv:1606.05162

[8] D. Girela, Logarithmic coefficients of univalent functions, Ann. Acad. Sci. Fenn. Math. 25 (2000), 337-350.

[9] A. W. Goodman, Univalent functions, Vol 1 (Tampa, Florida), Mariner Publishing, 1983.

[10] W. Kaplan, Close-to-convex schlicht functions, Michigan Math. J. 1(1952), 169-185.

[11] Z. Lewandowski, Sur l'identité de certaines classes de fonctions univalentes, I, Ann. Univ. Mariae CurieSkodowska Sect. A12 (1958), 131-146.

[12] R.J. Libera and E.J. Zlotkiewicz, Coefficient bounds for the inverse of a function with derivative in P-II, Proc. Amer. Math. Soc. 92 (1) (1984) 58-60.

[13] W. MA and D. Minda, A unified treatment of some special classes of univalent functions, Proc. Conf. on Complex Analysis, Tianjin, China, 1992, Proceeding and Lecture Notes in Analysis, 1 (International Press, 1994), 157-169

[14] C. Pommerenke, Univalent functions, Vandenhoeck \& Ruprecht, 1975.

[15] S. Ponnusamy, A. Vasudevarao and H. Yanaginara, Region of variability for close-toconvex functions, Complex Var. Elliptic Equ. 53(8)(2008), $709-716$.

[16] S. Ponnusamy, A. Vasudevarao and H. Yanagihara, Region of variability for close-toconvex functions-II, Appl. Math. Comput. 215(3)(2009), 901-915.

[17] Oliver Roth, A sharp inequality for the logarithmic coefficients of univalent functions, Proc. Amer. Math. Soc. 135(7) (2007), 2051-2054.

[18] D.K. Thomas, On the logarithmic coefficients of close to convex functions, Proc. Amer. Math. Soc. 144 (2016), 1681-1687.

[19] Z. Ye, The logarithmic coefficients of close-to-convex functions, Bulletin of the Institute of Mathematics Academia Sinica (New Series)32008, 445-452.

Pranav Kumar Upadrashta, Department of Mechanical Engineering, Indian Institute of Technology Kharagpur, Kharagpur-721 302, West Bengal, India.

E-mail address: upk1993@gmail.com

A. Vasudevarao, Department of Mathematics, Indian Institute of Technology Kharagpur, Kharagpur-721 302, West Bengal, India.

E-mail address: alluvasu@maths.iitkgp.ernet.in 